\title{
Inhaled Prostacyclin as Salvage Therapy for ARDS: Can We Find the Right Patient?
}

ARDS remains a clinically heterogeneous disorder wherein complex decision-making must be made when considering any salvage therapy. Inhaled nitric oxide and prostacyclin have emerged as an attempt to exploit the portions of the lung where tidal ventilation is preferentially distributed by inducing local pulmonary vasodilation and improving $\dot{V} / \mathbf{Q}$ matching. Both agents have shown improved oxygenation with their usage,,$^{1,2}$ although a mortality benefit has not been clearly demonstrated for either, ${ }^{3,4}$ and fewer data are available for inhaled prostacyclin when compared to nitric oxide. ${ }^{4}$ Given how heterogeneous a disorder ARDS is, more information is needed to help determine which subset of patients may benefit the most from this salvage therapy. For instance, previous studies of inhaled prostacyclin have shown that certain etiologies may benefit more when they are classified as direct versus indirect, ${ }^{5}$ while a study of inhaled nitric oxide noted decreased effectiveness when the underlying etiology was sepsis. ${ }^{6}$

In this issue of Respiratory CARE, Kallet et $\mathrm{al}^{7}$ sought to answer some of these ongoing questions. They specifically focused on analyzing who had the best response to inhaled prostacyclin by looking at surrogates for aerated parenchyma (ie, the functional residual capacity), including the $\mathrm{P}_{\mathrm{aO}} / \mathrm{F}_{\mathrm{IO}_{2}}$ ratio and the compliance of the respiratory system $\left(\mathrm{C}_{\mathrm{RS}}\right)$, as well as analyzing the underlying cause for ARDS and its classification (direct vs indirect).

In this large retrospective study of a hospital's ARDS quality-assurance database spanning 14 years (from June 2002 to April 2016), 279 subjects received aerosolized prostacyclin (PGI2). After excluding those who died during therapy, had intervening increases in PEEP, recruitment maneuvers, prone positioning, or had missing data, there remained 208 subjects who had an arterial blood gas prior to and after initiation of PGI2 available for analysis. All subjects underwent ARDSnet ventilatory protocols. The subjects were reclassified post hoc according to the current Berlin definition of ARDS. Logistic regression was

The authors have disclosed no conflicts of interest.

Correspondence: Amy H Attaway MD, Division of Pulmonary \& Critical Care Medicine, University Hospitals Cleveland, 11100 Euclid Avenue, Cleveland, OH 44106. E-mail: amyattaway25@gmail.com.

DOI: $10.4187 /$ respcare. 05708 used to determine which variables predicted PGI2 responders (defined as a $>10 \mathrm{~mm} \mathrm{Hg}$ increase in $\mathrm{P}_{\mathrm{aO}_{2}}$ ) from nonresponders, which is based on a study by Walmrath et al. ${ }^{8}$

See the Original Study on Page 1014

Not surprisingly, the subjects from this retrospective study of an ARDS salvage therapy were very ill. The mean APACHE II (Acute Physiology and Chronic Health Evaluation) score was 26.2 and SAPS II (Simplified Acute Physiology Score) was 53. Baseline $\mathrm{P}_{\mathrm{aO}_{2}} / \mathrm{F}_{\mathrm{IO}_{2}}$ was classified as $<60 \mathrm{~mm} \mathrm{Hg}$ (representing $59 \%$ at study onset and $83 \%$ at initiation), $60-90 \mathrm{~mm} \mathrm{Hg}$ (35\% at onset, $16 \%$ at initiation), and $>90 \mathrm{~mm} \mathrm{Hg}$ (5\% at onset, $1 \%$ at initiation). Mean in-hospital mortality was $56 \%$ for the subjects included in the analysis. When adding in the 12 patients who died during initiation of therapy, the overall mortality for this study approaches $60 \%$, which is higher than most ARDS studies 9,10 and makes it more difficult to extrapolate this population to others. Overall, inhaled PGI2 demonstrated an increased $\mathrm{P}_{\mathrm{aO}_{2}} / \mathrm{F}_{\mathrm{IO}_{2}}$ ratio by $33 \mathrm{~mm} \mathrm{Hg}$ for the entire cohort and by $56 \mathrm{~mm} \mathrm{Hg}$ when only responders were considered.

The magnitude of this is consistent with other studies, although higher than others. ${ }^{11}$ Of those who responded, subjects tended to be less sick with a higher $\mathrm{P}_{\mathrm{aO}_{2}} / \mathrm{F}_{\mathrm{IO}_{2}}$ ratio at onset. When only looking at responders, the lowest $\mathrm{P}_{\mathrm{aO}_{2}} / \mathrm{F}_{\mathrm{IO}_{2}}$ group improved by $39 \pm 45 \mathrm{~mm} \mathrm{Hg}(P<.001)$, the median group by $58 \pm 46(P<.05)$, and the highest group improved by $70 \pm 49(P<.05)$. The authors emphasized the concept of responders versus non-responders, which is one way to reconcile the clinical relevance of smaller mean improvement but larger improvement in select populations. However, in the absence of mortality data, this brings into question its relevance. On a practical basis, $<10 \mathrm{~mm} \mathrm{Hg}$ improvement logically appears to be of little value in any clinical setting; however, one might argue that marginal improvements of 10-20 $\mathrm{mm} \mathrm{Hg}$ may be of little value in patients with a $\mathrm{P}_{\mathrm{aO}_{2}}$ of $>60-70$.

In terms of the etiology for ARDS, those with trauma had the biggest increase in $\mathrm{P}_{\mathrm{aO}} / \mathrm{F}_{\mathrm{IO}_{2}}$ ratio, while those with sepsis had the least. The average improvement for sepsis was $18 \pm 35 \mathrm{~mm} \mathrm{Hg}$ compared with $40 \pm 55 \mathrm{~mm} \mathrm{Hg}$ for non-sepsis etiologies $(P=.002)$, and only $50 \%$ of sepsis subjects were responders. These findings are consistent 
with other studies of inhaled nitric oxide ${ }^{6,12}$ and are postulated to be due to dysregulated release of nitric oxide and prostacyclin. ${ }^{13}$ The magnitude of improvement for direct, indirect, and mixed ARDS was $34 \pm 51,22 \pm 45$, and $41 \pm 51 \mathrm{~mm} \mathrm{Hg}$, respectively. Significance was seen only when comparing the indirect and mixed mechanisms $(P<.05)$. In addition, the responder rates ranged from $55-67 \%$ and were not significantly different across groups. Ultimately, classifying ARDS as direct, indirect, or mixed did not help in predicting responder rate or the magnitude of improvement as previous studies have done. ${ }^{12}$

While the initial $\mathrm{P}_{\mathrm{aO}_{2}} / \mathrm{F}_{\mathrm{IO}_{2}}$ ratio predicted proportional increases in oxygenation after inhaled PGI2 therapy, $\mathrm{C}_{\mathrm{RS}}$ did not demonstrate the same pattern consistently across categories. Nevertheless, the authors noted that, as the $C_{R S}$ improved, it predicted responders to inhaled prostacyclin.

In multivariate regression analysis, the 2 major predictors for response to inhaled PGI2 were the $\mathrm{P}_{\mathrm{aO}_{2}} / \mathrm{F}_{\mathrm{IO}}$ ratio $(\mathrm{OR} 1.10, P=.042)$ and $\mathrm{C}_{\mathrm{RS}}(\mathrm{OR} 1.04, P=.02)$. The clinical relevance of these predictors in the absence of mortality analysis or surrogates like the need for extracorporeal membrane oxygenation is harder to interpret. The authors posited that the effectiveness of aerosolized PGI2 is dependent on aerated lung parenchyma (or functional residual capacity) as predicted by surrogate measures like the $\mathrm{P}_{\mathrm{aO}} / \mathrm{F}_{\mathrm{IO}_{2}}$ ratio and $\mathrm{C}_{\mathrm{RS}}$ and therefore should be combined with other strategies to recruit lung parenchyma, such as higher PEEP, prone positioning, and recruitment maneuvers. This is consistent with recent observational studies combining prone positioning and inhaled nitric oxide showing an additive effect of oxygenation. ${ }^{14,15}$ Prone positioning itself has been shown to increase the number of responders to nitric oxide. ${ }^{15}$ While we agree with the authors on this concept, we would like to highlight an additional point on recruitment maneuvers.

While the current literature favors higher PEEP and prone positioning as showing improved mortality outcomes for severe ARDS, ${ }^{16,17}$ the data on recruitment maneuvers for ARDS are somewhat mixed. While there are data to support the major methods of recruitment maneuvers (which include sustained, decremental, and stepwise) in the postoperative setting after general anesthesia, sustained and decremental recruitment methods for ARDS have remained controversial. Of primary concern is the relative hemodynamic instability that can result secondary to abrupt increases in intrathoracic pressure, which can lead to decreased cardiac output followed by hypotension and desaturation. ${ }^{18,19}$ For this reason, a stepwise approach has been favored for ARDS utilizing incremental PEEP titration, with PEEP being gradually increased and changes occurring every $3-5$ min. ${ }^{18}$

The difficulty in reaching a consensus on the use of recruitment maneuvers is likely due to the large heterogeneity of patient populations as well as variability in tech- niques. Much of the evidence that currently exists focuses on physiologic assessments rather than outcomes. By measuring negative responses to the maneuvers such as hypotension, desaturation, and increased plateau pressure, some argue that the maneuvers may be unsafe and cause detrimental effects. Additionally, of the studies including recruitment maneuvers that do demonstrate improvements in mortality, often other recruitment strategies known to improve outcomes (eg, high PEEP, prone positioning) were being used as well. ${ }^{20}$ Another important point to highlight is that certain patients seem to respond to lung recruitment while others do not, which adds to the complexity of this process and may be why consistent clinical evidence remains elusive. ${ }^{21}$ The results of ongoing large, multi-center randomized control studies ${ }^{22,23}$ may shed more light on this subject in the future.

Salvage therapy for ARDS is generally defined as additional measures beyond ARDSnet ventilator strategies employed for patients who have deficits in oxygenation despite maximum ventilatory strategies where $\mathrm{P}_{a}$ may become limiting and life-threatening. This study clearly gives us more important clinical information on real-world use of inhaled prostacyclin. It is not clear on the basis of this study, however, that we can include or exclude consideration of ARDS patients for this particular salvage technique. It seems that the patients most desperately in need of help appear to be the least likely to improve, and those with the most robust improvement did not appear to have an initial $\mathrm{P}_{\mathrm{aO}_{2}}$ that was life-threatening in the first place.

The heterogeneity of ARDS and its complex management strategies clearly complicate evaluation of salvage therapies. The critical end point is mortality, but, in the interim, evaluation of important surrogate end points such as significant reductions in administered $\mathrm{F}_{\mathrm{IO}_{2}}$, decrease in the use or intensity of other salvage therapies, or the need for extracorporeal membrane oxygenation may add important information. Another useful end point may be identifying patients with very little chance of meaningful physiologic improvement where therapy would not be considered a meaningful option. It is hard to clearly outline a population from the data provided by this study, although the authors' emphasis on improving the effectiveness of inhaled prostacyclin by increasing aerated lung parenchyma through higher PEEP, prone positioning, and recruitment maneuvers remain important therapeutic aspects of ARDS. At this time, however, we are still limited in our ability to confidently find the right patient.

\footnotetext{
Amy H Attaway MD Catherine Myers MD Shrey Velani MD Robert Schilz DO PhD University Hospital Cleveland Cleveland Ohio
} Department of Pulmonary and Critical Care Medicine 


\section{EDITORIALS}

\section{REFERENCES}

1. Bigatello LM, Hurford WE, Kacmarek RM, Roberts JD, Zapol WM. Prolonged inhalation of low concentrations of nitric oxide in subjects with severe adult respiratory distress syndrome. Anesthesiology 1994; 80(4):761-770.

2. Walmrath D, Schneider T, Pilch J, Grimminger F, Seeger W. Aerosolized prostacyclin in adult respiratory distress syndrome. Lancet 1993;342(8877):961-962.

3. Adhikari NK, Dellinger RP, Lundin S, Payen D, Vallet B, Gerlach $\mathrm{H}$, et al. Inhaled nitric oxide does not reduce mortality in patients with acute respiratory distress syndrome regardless of severity: systematic review and meta-analysis. Crit Care Med 2014;42(2):404412.

4. Dzierba AL, Abel EE, Buckley MS, Lat I. A review of inhaled nitric oxide and aerosolized epoprostenol in acute lung injury or acute respiratory distress syndrome. Pharmacotherapy 2014 Mar;34(3):279290.

5. Domenighetti G, Stricker H, Waldispuehl B. Nebulized prostacyclin (PGI2) in acute respiratory distress syndrome: Impact of primary (pulmonary injury) and secondary (extrapulmonary injury) disease on gas exchange response. Crit Care Med 2001;29(1):57-62.

6. Manktelow C, Bigatello LM, Hess D, Hurford WE. Physiologic determinants of the response to inhaled nitric oxide in subjects with acute respiratory distress syndrome. Anesthesiology 1997;87(2):297307.

7. Kallet R, Burns G, Zhuo H, Ho K, Phillips J, Pangilinan L, et al. Severity of hypoxemia and other factors that influence the response to aerosolized prostacyclin in ARDS. Respir Care 2017;62(8):10141022.

8. Walmrath D, Schneider T, Schermuly R, Olschewski H, Grimminger F, Seeger W. Direct comparison of inhaled nitric oxide and aerosolized prostacyclin in acute respiratory distress syndrome. Am J Respir Crit Care Med 1996;153(3):991-996.

9. The ARDS Definition Taskforce. Acute respiratory distress syndrome: the Berlin definition. JAMA 2012;307(23):2526-2533.

10. Bellani G, Laffey JG, Fan E, Brochard L, Esteban A, Gattinoni L, et al. Epidemiology, patterns of care and mortality for patients with acute respiratory distress syndrome in intensive care untis in 50 countries. JAMA 2016;315(8):788-800.

11. Fuller BM, Mohr NM, Skrupky L, Fowler S, Kollef MH, Carpenter CR. The use of inhaled prostaglandins in patients with ARDS: a systematic review and meta-analysis. Chest 2015 Jun;147(6):151022.

12. Krafft P, Fridrich P, Fitzgerald RD, Koc D, Steltzer H. Effectiveness of nitric oxide inhalation in septic ARDS. Chest 1996;109(2):486493.

13. Boisrame-Helms J, Kremer H, Schini-Kerth V, Meziani F. Endothelial dysfunction in sepsis. Curr Vasc Pharmacol 2013;11(2):150-160.

14. Papazian L, Bregeon F, Gaillat F, Thirion X, Gainnier M, Gregoire $\mathrm{R}$, et al. Respective and combined effects of prone position and inhaled nitric oxide in patients with acute respiratory distress syndrome. Am J Respir Crit Care Med 1998;157(2):580-585.

15. Martinez M, Diaz E, Joseph D, Villagrá A, Mas A, Fernandez R, Blanch L. Improvement in oxygenation by prone position and nitric oxide in patients with acute respiratory distress syndrome. Intensive Care Med 1999;25(1):29-36.

16. Guérin C, Reignier J, Richard JC, Beuret P, Gacouin A, Boulain T, et al. Prone positioning in severe acute respiratory distress syndrome. N Engl J Med 2013;368(23):2159-2168.

17. Briel M, Meade M, Mercat A, Brower RG, Talmor D, Walter SD, et al. Higher vs lower positive end-expiratory pressure in patients with acute lung injury and acute respiratory distress syndrome: systematic review and meta-analysis. JAMA 2010;303(9):865-873.

18. Hess DR. Recruitment maneuvers and PEEP titration. Respir Care 2015;60(11):1688-1704.

19. Das A, Haque M, Chikhani M, Cole O, Wang W, Hardman JG, Bates DG. Hemodynamic effects of lung recruitment maneuvers in acute respiratory distress syndrome. BMC Pulm Med 2017;17(1):34.

20. Hodgson C, Goligher EC, Young ME, Keating JL, Holland AE, Romero L, et al. Recruitment manoeuvres for adults with acute respiratory distress syndrome receiving mechanical ventilation. Cochrane Database Syst Rev 2016;(11):CD006667.

21. Gattinoni L, Caironi P, Cressoni M, Chiumello D, Ranieri VM, Quintel M, et al. Lung recruitment in patients with the acute respiratory distress syndrome. N Engl J Med 2006;354(17):1775-1786.

22. ART Investigators. Rationale, study design, and analysis plan of the Alveolar Recruitment for ARDS Trial (ART): study protocol for a randomized controlled trial. Trials 2012;13:153.

23. A multi-centre trial of an open lung strategy including permissive hypercapnia, alveolar recruitment and low airway pressure in patients with acute respiratory distress syndrome (PHARLAP). (2012). https://www.clinicaltrials.gov (Identification No. NCT01667146). Accessed June 8, 2017. 